\title{
A Modified Compressed Sensing-based Recovery Algorithm for Wireless Sensor Networks
}

\author{
Javad Afshar JAHANSHAHI, Habibollah DANYALI, Mohammad Sadegh HELFROUSH
}

Dept. of Electrical and Electronics Engineering, Shiraz University of Technology, Shiraz, Iran

\{J.afshar, Danyali, Ms_helfroush\}@ sutech.ac.ir

Submitted November 21, 2018 / Accepted July 9, 2019

\begin{abstract}
In this paper, a novel compressed sensing (CS) acquisition and joint recovery of spatiotemporal correlated signals algorithm is presented for effective data collection and precise sensors data streams reconstruction in wireless sensor networks. The CS-based proposed method utilizes an iterative re-weighted $\ell_{1}$-minimization and a $\ell_{2}$ regularization to increase the reconstruction accuracy with a small number of required data transmission. Moreover, we develop an alternating direction method of multipliers based algorithm to efficiently solve the resulting optimization problem. Numerical experiments are conducted on several test signals with a variety of sampling ratios. The experimental results verify the effectiveness of the proposed scheme in terms of reconstruction accuracy and consumption time compared with the state of the art algorithms.
\end{abstract}

\section{Keywords}

Distributed compressive sensing (DCS), regularization, iterative re-weighted $\ell_{1}$-minimization, wireless sensor networks

\section{Introduction}

The advances in the field of telecommunication and newly developed applications have increased the need for deploying distributed wireless sensor networks (WSNs), which of multiple sensors for monitoring, sensing, collecting, processing and transmitting specific data to a sink for signal recovery in an area of interest. Due to the main challenges of the energy constraint WSNs, reducing the overall energy consumption of the sensor nodes is of great importance. In this respect, compressive sensing (CS) has drawn much interest, simultaneously providing a sensing and compression framework [1-9], enabling a potentially significant reduction in the sampling and computational costs of a sensor with limited capabilities; while still guaranteeing perfect recovery [10-13]. Although CS has established a promising performance in exploiting the spatial or temporal correlation, the necessary data transmission can be decreased more.
As the physical phenomenon monitored data from neighboring sensors encompass spatiotemporal correlation, the higher cost data transmission can be optimized via distributed data compression/acquisition techniques [14]. Recently, the theory of distributed compressive sensing (DCS) [15] have been used to exploit spatiotemporal correlations via joint sparsity models [16]. A spatiotemporal correlation-based distributed compressive sensing algorithm which exploits the belief propagation method is presented in [17] to reconstruct the original signal. In order to obtain a better reconstruction performance, a PCA based algorithm is proposed in [18] via exploiting the correlation characteristics among data. Since these methods utilize the correlation amongst the sensors' readings, they can not achieve high-level accuracy with lower sampling ratio. Therefore, an efficient iterative re-weighted recovery algorithm [19] employed a sliding data window not only to successively exploit overlapping signals but also improve the reconstruction accuracy with fast convergence. Afterward, a CS based algorithm was proposed to improve the recovery performance of the spatio-temporal correlated sensor data streams [20].

In order to improve the recovery performance of the gathered sensors' readings for the real-time WSN applications, a DCS based algorithm is proposed in this paper as an extension of the iterative re-weighted $\ell_{1}$ minimization algorithm $\left(\right.$ IRW $\left.-\ell_{1}\right)$ [21] and joint recovery algorithm in [22]. Inspired from [20], the proposed DCS based algorithm is formulated in the form of a minimization function under a regularization-based framework. Based on the alternating direction method of multipliers (ADMM) algorithm [23], an efficient, relatively fast and ease of implementation alternating minimization approach is developed to solve the regularization based minimization algorithm. The simulation results verify that the proposed framework substantially outperform different conventional and state of the art techniques in terms of recovery accuracy and optimization problem solving time.

The rest of this paper is organized as follows: In Sec. 2, the whole scenario is presented in detail to adaptively measure and reconstruct the sparse signal ensemble. In Sec. 3, the performance of the proposed method is compared with the state of the art algorithms. Finally, the paper concludes in Sec. 4. Throughout this paper, vectors are denoted in bold 
lowercase letters, matrices are written in bold uppercase letters, ()$^{\mathrm{T}}$ and ()$^{\mathrm{H}}$ denote the vector and matrix transpose and Hermitian operators, respectively. ()$^{-1}$ stands for the inverse operation and the inner product is denoted by $\langle$,$\rangle .$

\section{System Model}

For the simplicity of analysis, consider a single-hop wireless sensor network with $J$ sensors and one sink in which sensors are distributed at a number of outdoor locations measuring an event such as temperature, pressure, wind speed, etc. throughout the day. Let $\mathbf{X}(\tau) \in R^{J \times L}$ consist of $L$ successive readings of all $J$ sensor nodes at equal-sized time slot $\tau \in\{\tau-L+1, \ldots, \tau\}$ as

$$
\mathbf{X}(\tau)=\left[\begin{array}{ccc}
x_{1}(\tau-L+1) & \cdots & x_{1}(\tau) \\
\vdots & \ddots & \vdots \\
x_{J}(\tau-L+1) & \cdots & x_{J}(\tau)
\end{array}\right]
$$

where $x_{j}(\tau)$ is the reading of $j$ th sensor at time instant $\tau$. Let us denote the the rows of $\mathbf{X}(\tau)$ contain the reading of all sensors $j \in J$ at time instants $\tau \in\{\tau-L+1, \ldots, \tau\}$ and the columns contain the sensors' readings at time instant $\tau$. Therefore, $\mathbf{X}(\tau)$ can be expressed as

$$
\mathbf{X}(\tau)=\left[x_{1}(\tau) \cdots x_{J}(\tau)\right]^{\mathrm{T}}=[\bar{x}(\tau-L+1) \cdots \bar{x}(\tau)]
$$

As the joint sparsity model described in [15], all sensor readings share a common component $\mathbf{x}_{c}(\tau) \in R^{L}$ such that $\mathbf{x}_{j}(\tau)=\mathbf{x}_{c}(\tau)+\mathbf{x}_{\mathrm{in}_{j}}(\tau),(j \in 1,2, \ldots, J)$, where $\mathbf{x}_{\mathrm{in}_{j}}(\tau) \in R^{L}$ is the innovation part of each signal $\mathbf{x}_{j}(\tau)$. There is a dictionary $D \in R^{L \times K}$ that sparsely represents the signal $\left(\mathbf{x}_{j}(\tau)=D \boldsymbol{\alpha}_{j}(\tau)=D\left(\boldsymbol{\alpha}_{c}(\tau)+\boldsymbol{\alpha}_{\mathrm{in}_{j}}(\tau)\right)\right)$ where $\left|\boldsymbol{\alpha}_{c}(\tau)\right|_{\ell_{0}}=k_{c}$ and $\left|\boldsymbol{\alpha}_{\mathrm{in}_{j}(\tau)}\right|_{\ell_{0}}=k_{j}$.

In each sensor a CS encoding procedure is considered, where at each time instant $\tau \geq 1$, the measurements are taken with respect to the current sensors' reading $\bar{x}(\tau)$. Thus, as the data gathering algorithm presented in [24], the sink will be sent $J$ linear CS measurements as

$$
\begin{aligned}
& {\left[\begin{array}{c}
\bar{r}(\tau-L+1) \\
\vdots \\
\bar{r}(\tau)
\end{array}\right]=} \\
& {\left[\begin{array}{ccc}
\bar{\Phi}(\tau-L+1) & \cdots & 0 \\
\vdots & \ddots & \vdots \\
0 & \cdots & \bar{\Phi}(\tau)
\end{array}\right]\left[\begin{array}{c}
\bar{x}(\tau-L+1) \\
\vdots \\
\bar{x}(\tau)
\end{array}\right]}
\end{aligned}
$$

Accordingly, by reforming the measurements $\mathbf{R}(\tau)=$ $\left[\bar{r}^{\mathrm{T}}(\tau-L+1) \cdots \bar{r}^{\mathrm{T}}(\tau)\right]^{\mathrm{T}} \in R^{\sum_{t=\tau-L+1}^{\tau} M_{\mathbf{x}_{j}(t)}}$, and the measurement matrix $\Phi(\tau)=\operatorname{diag}(\bar{\Phi}(\tau-L+1) \cdots \bar{\Phi}(\tau)) \in$
$R^{\sum_{t=\tau-L+1}^{\tau} M_{\mathbf{x}_{j}(t) \times J L}}$, the measurement ensemble in (3) can be compactly rewritten as $\mathbf{R}(\tau)=\Phi(\tau) x(\tau)$ where $x(\tau)=$ $\operatorname{vec}(\mathbf{X}(\tau))$. Note that the measurement matrices $\bar{\Phi}(\tau) \in$ $R^{M_{\mathbf{x}_{j}(t)}} \times J$ have varying number of measurements $M_{\mathbf{x}_{j}(t)}$ that depend on the sparsity order of the signals.

Obviously, $\mathbf{R}(\tau)=\left[r_{1}(\tau) \cdots r_{J}(\tau)\right]^{\mathrm{T}} \in R^{\sum_{t=\tau-L+1}^{\tau} M_{\mathbf{x}_{j}(t)}}$ where $\mathbf{r}_{j}(\tau)$ is the $j$ th measured signals in the sink that consists of two parts: the common part $\mathbf{r}_{c_{j}}(\tau) \in R^{M_{\mathbf{x}_{j}}(\tau)}$ and the innovation part $\mathbf{r}_{\mathrm{in}_{j}}(\tau) \in R^{M_{\mathbf{x}_{j}}(\tau)}$, as follows:

$$
\begin{gathered}
\mathbf{r}_{j}(\tau)=\mathbf{r}_{c_{j}}(\tau)+\mathbf{r}_{\mathrm{in}_{j}}(\tau) \\
\mathbf{r}_{c_{j}}(\tau)=\Phi_{j}(\tau) \mathbf{x}_{c}(\tau), \mathbf{r}_{i n_{j}}(\tau)=\Phi_{j}(\tau) \mathbf{x}_{\mathrm{in}_{j}}(\tau)
\end{gathered}
$$

Inspired from JSM models [15], the problem to recover the signal ensemble of the sensor nodes can be expressed as solving an $\ell_{1}$ minimization problem as follows:

$$
\hat{\boldsymbol{\alpha}}(\tau):=\arg \min _{\hat{\boldsymbol{\alpha}}(\tau)}|\dot{\boldsymbol{\alpha}}(\tau)|_{\ell_{1}}, \text { s.t. } \mathbf{R}(\tau)=\Phi(\tau) \Psi \dot{\boldsymbol{\alpha}}(\tau)
$$

where $\hat{\boldsymbol{\alpha}}(\tau)=\left[\begin{array}{llll}\hat{\boldsymbol{\alpha}}_{c}^{\mathrm{T}}(\tau) & \hat{\boldsymbol{\alpha}}_{\mathrm{in}_{1}}^{\mathrm{T}}(\tau) & \cdots & \hat{\boldsymbol{\alpha}}_{\mathrm{in}_{J}}^{\mathrm{T}}(\tau)\end{array}\right]^{\mathrm{T}}$ and the dictionary matrices $\boldsymbol{\Psi}$ is as follows:

$$
\boldsymbol{\Psi}=\left[\begin{array}{ccccc}
D & D & 0 & \cdots & 0 \\
D & 0 & D & \cdots & \vdots \\
\vdots & & & \ddots & 0 \\
D & 0 & \cdots & 0 & D
\end{array}\right]
$$

Then, $\hat{\mathbf{x}}_{j}(\tau)$ is computed by following:

$$
\hat{\mathbf{x}}_{j}(\tau)=D\left(\hat{\boldsymbol{\alpha}}_{c}(\tau)+\hat{\boldsymbol{\alpha}}_{\mathrm{in}_{j}}(\tau)\right)
$$

where $\hat{\boldsymbol{\alpha}}_{c}(\tau)$ and $\hat{\boldsymbol{\alpha}}_{\mathrm{in}_{j}}(\tau) \mathrm{s}$ are within the obtained $\hat{\boldsymbol{\alpha}}(\tau)$ vector.

\subsection{Development of the Reconstruction Algorithm}

In this subsection, we are going to improve the reconstruction accuracy and time consumed by the proposed method. In this sense, assuming that sink knows the common part $\boldsymbol{\alpha}_{c}(\tau)$ of the transmitted signals for each time slot $\tau$, then $\boldsymbol{\alpha}_{c}(\tau)$ is omitted from the reconstruction of (5) by the following mathematical ways [22]:

$$
\mathbf{R}(\tau)=[\Phi(\tau) \Psi]\left[\begin{array}{c}
\boldsymbol{\alpha}_{c}(\tau) \\
\boldsymbol{\alpha}_{\mathrm{in}_{1}}(\tau) \\
\vdots \\
\boldsymbol{\alpha}_{\mathrm{in}_{J}}(\tau)
\end{array}\right]=[A(\tau) \| H(\tau)]\left[\begin{array}{c}
\boldsymbol{\alpha}_{c}(\tau) \\
- \\
\boldsymbol{\alpha}_{\mathrm{in}_{1}}(\tau) \\
\vdots \\
\boldsymbol{\alpha}_{\mathrm{in}_{J}}(\tau)
\end{array}\right]
$$


Equation (8) can be rewritten as follows:

$$
\mathbf{R}(\tau)=A(\tau) \boldsymbol{\alpha}_{c}(\tau)+H(\tau) \boldsymbol{\alpha}_{I}(\tau)
$$

where $A(\tau)$ and $H(\tau)$ are combination matrix joining of the different involved parameters and $\boldsymbol{\alpha}_{I}(\tau)=$ $\left[\boldsymbol{\alpha}_{\mathrm{in}_{1}}(\tau)^{\mathrm{T}} \boldsymbol{\alpha}_{\mathrm{in}_{2}}(\tau)^{\mathrm{T}} \cdots \boldsymbol{\alpha}_{\mathrm{in}_{J}}(\tau)^{\mathrm{T}}\right]^{\mathrm{T}}$.

Hence, a weighting parameter is assigned to the coefficients, which is inversely proportional to the expected coefficients magnitude. Then, we incorporate the iterative re-weighted $\ell_{1}$ minimization algorithm (IRW- $\ell_{1}$ ) [21] to the corresponding modified unconstrained problem (5) as follows:

$$
\begin{aligned}
\hat{\boldsymbol{\alpha}}_{I}^{(n)}(\tau):=\arg \min _{\boldsymbol{\alpha}_{I}^{\prime}(\tau)} \eta_{1}\left|W^{(n)}(\tau) \boldsymbol{\alpha}_{I}^{\prime}(\tau)\right|_{\ell_{1}} \\
+\frac{1}{2}\left|\mathbf{R}_{I}(\tau)-H(\tau) \hat{\boldsymbol{\alpha}}_{I}^{(n)}(\tau)\right|_{\ell_{2}}^{2}
\end{aligned}
$$

where $\mathbf{R}_{I}(\tau)=\mathbf{R}(\tau)-A(\tau) \boldsymbol{\alpha}_{c}(\tau)$. In addition, we adapt $\ell_{2}$-norm regularization $\eta_{2}\left|\hat{x}^{(n)}(\tau)-\hat{x}^{(n-1)}(\tau)\right|_{\ell_{2}}^{2}$ for the variations in the estimates $\hat{x}^{(n)}(\tau)$ and $\hat{x}^{(n-1)}(\tau)$ obtained at the successive recovery instants as of [20]. Then, the sparsity constrain and the variations in the estimates are incorporated by minimizing the IRW- $\ell_{1}$ and the $\ell_{2}$-norm regularization in a single formulation as follows:

$$
\begin{aligned}
& \hat{\boldsymbol{\alpha}}_{I}^{(n)}(\tau):=\arg \min _{\boldsymbol{\alpha}_{I}(\tau)} \eta_{1}\left|W^{(n)}(\tau) \boldsymbol{\alpha}_{I}^{\prime}(\tau)\right|_{\ell_{1}} \\
+ & \frac{1}{2}\left|\mathbf{R}_{I}(\tau)-H(\tau) \hat{\boldsymbol{\alpha}}_{I}^{(n)}(\tau)\right|_{\ell_{2}}^{2}+\frac{\eta_{2}}{2}\left|\hat{x}^{(n)}(\tau)-\hat{x}^{(n-1)}(\tau)\right|_{\ell_{2}}^{2}
\end{aligned}
$$

where the regularization weight parameters $\eta_{1}$ and $\eta_{2} \geq 0$ control the trade-off between the $\ell_{2}$-norm regularization and sparsity feature $\ell_{1}$-norm of the data from current time slot, and

$$
\begin{aligned}
\hat{x}^{(n)}(\tau) & =\boldsymbol{\Psi}\left[\hat{\boldsymbol{\alpha}}_{c}^{(n) T}(\tau) \hat{\boldsymbol{\alpha}}_{\mathrm{in}_{1}}^{(n) T}(\tau) \cdots \hat{\boldsymbol{\alpha}}_{\mathrm{in}_{J}}^{(n) T}(\tau)\right]^{\mathrm{T}} \\
& =\boldsymbol{\Psi}\left[\hat{\boldsymbol{\alpha}}_{c}^{(n) T}(\tau) \hat{\boldsymbol{\alpha}}_{I}^{(n) T}(\tau)\right]^{\mathrm{T}} .
\end{aligned}
$$

Then, in order to update the weights $W^{(n+1)}$, the obtained vector $\hat{\boldsymbol{\alpha}}_{I}^{(n)}(\tau)$ is used as [21]:

$$
\left(w_{i}\right)^{(n+1)}(\tau)=\left(\hat{\boldsymbol{\alpha}}_{\mathrm{in}_{i}}^{(n)}(\tau)+\epsilon_{0}\right)^{-1}, i=1, \cdots, L J
$$

where $\epsilon_{0}>0$ is a small parameter and $W^{(n)}(\tau)=$ $\operatorname{diag}\left(\left(w_{1}\right)^{(n)}(\tau),\left(w_{2}\right)^{(n)}(\tau), \cdots,\left(w_{L J}\right)^{(n)}(\tau)\right)$. The algorithm is terminated when $\left|W^{(n+1)}(\tau)-W^{(n)}(\tau)\right|_{\ell_{2}} /\left|W^{(n)}(\tau)\right|_{\ell_{2}}$ is smaller than a pre-defined threshold or $n=n_{\max }$.

\subsection{A Fast and Efficient Analytical Solution for the IRW $-\ell_{1}$ Based Minimization Algorithm}

Although the IRW- $\ell_{1}$ based minimization algorithm in (11) is a convex optimization problem and the imposed constraints are appealing from a modeling standpoint, it is acknowledged that solving it using methods such as interior points algorithms raises issue of computational complexity to running time of $O\left(N^{3}\right)$ [25]. In this paper, we employ a developed method based on the alternating direction method of multipliers (ADMM) algorithm [23] due to its suitability in efficiently solving the large-scale convex optimization problems, ease of implementation and relatively fast. For this purpose, the optimization problem in (11) is converted into the following equivalent constrained-form through variable splitting as follows (for the simplicity the subscript $(n)$ and $(\tau)$ are omitted without confusion):

$$
\begin{aligned}
\left\{\hat{\boldsymbol{\alpha}}_{I}, \hat{\mathfrak{J}}\right\} & =\underset{\hat{\boldsymbol{\alpha}}_{I}, \hat{\mathfrak{J}}}{\arg \min } \eta_{1}|\hat{\mathfrak{J}}|_{\ell_{1}}+\frac{1}{2}\left|\mathbf{R}_{I}-H \hat{\boldsymbol{\alpha}}_{I}\right|_{\ell_{2}}^{2} \\
& +\frac{\eta_{2}}{2}\left|\boldsymbol{\Psi}\left[\hat{\boldsymbol{\alpha}}_{c}^{\mathrm{T}} \hat{\boldsymbol{\alpha}}_{I}^{\mathrm{T}}\right]^{\mathrm{T}}-\hat{x}^{B}\right|_{\ell_{2}}^{2} \text { s.t. } \hat{\mathfrak{J}}=W \hat{\boldsymbol{\alpha}}_{I} .
\end{aligned}
$$

Therefore, the augmented Lagrangian function for (14) is written as follows:

$$
\begin{gathered}
\mathcal{L}\left(\hat{\boldsymbol{\alpha}}_{I}, \hat{\mathfrak{J}}, \beta\right)=\frac{1}{2}\left|\mathbf{R}_{I}-H \hat{\boldsymbol{\alpha}}_{I}\right|_{\ell_{2}}^{2}+|\hat{\mathfrak{J}}|_{\ell_{1}}+\left\langle\beta, \hat{\mathfrak{J}}-W \hat{\boldsymbol{\alpha}}_{I}\right\rangle \\
+\frac{\delta}{2}\left|\hat{\mathfrak{J}}-W \hat{\boldsymbol{\alpha}}_{I}\right|_{\ell_{2}}^{2}+\frac{\eta_{2}}{2}\left|\boldsymbol{\Psi}\left[\begin{array}{ll}
\hat{\boldsymbol{\alpha}}_{c}^{\mathrm{T}} & \hat{\boldsymbol{\alpha}}_{I}^{\mathrm{T}}
\end{array}\right]^{\mathrm{T}}-\hat{x}^{B}\right|_{\ell_{2}}^{2}
\end{gathered}
$$

where $\beta$ is the Lagrangian multiplier, and $\delta$ is the penalty parameter related to convergence speed of the algorithm. In order to find the minimization of (15), the following alternating direction method is utilized as:

$$
\begin{aligned}
& \hat{\boldsymbol{\alpha}}_{I}^{k+1}=\arg \min _{\hat{\boldsymbol{\alpha}}_{I}} \mathcal{L}\left(\hat{\boldsymbol{\alpha}}_{I}, \hat{\mathfrak{I}}^{k}, \beta^{k}\right), \\
& \hat{\mathfrak{J}}^{k+1}=\arg \min _{\hat{\mathfrak{I}}} \mathcal{L}\left(\hat{\boldsymbol{\alpha}}_{I}^{k+1}, \hat{\mathfrak{J}}, \beta^{k}\right), \\
& \beta^{k+1}=\beta^{k}+\delta\left(\hat{\mathfrak{J}}^{k+1}-W \hat{\boldsymbol{\alpha}}_{I}^{k+1}\right) .
\end{aligned}
$$

The sub-problem of (16) can be rewritten as:

$$
\begin{array}{r}
\hat{\boldsymbol{\alpha}}_{I}^{k+1}=\arg \min _{\hat{\boldsymbol{\alpha}}_{I}} \frac{1}{2}\left|\mathbf{R}_{I}-H \hat{\boldsymbol{\alpha}}_{I}\right|_{\ell_{2}}^{2}+\left\langle\beta^{k}, \hat{\mathfrak{J}}^{k}-W \hat{\boldsymbol{\alpha}}_{I}\right\rangle \\
+\frac{\delta}{2}\left|\hat{\mathfrak{I}}^{k}-W \hat{\boldsymbol{\alpha}}_{I}\right|_{\ell_{2}}^{2}+\frac{\eta_{2}}{2}\left|\boldsymbol{\Psi}\left[\hat{\boldsymbol{\alpha}}_{c}^{\mathrm{T}} \hat{\boldsymbol{\alpha}}_{I}^{\mathrm{T}}\right]^{\mathrm{T}}-\hat{x}^{B}\right|_{\ell_{2}}^{2} \\
=\arg \min _{\hat{\boldsymbol{\alpha}}_{I}} \frac{1}{2}\left|\mathbf{R}_{I}-H \hat{\boldsymbol{\alpha}}_{I}\right|_{\ell_{2}}^{2}+\frac{\delta}{2}\left|\hat{\mathfrak{J}}^{k}-W \hat{\boldsymbol{\alpha}}_{I}+\frac{\beta^{k}}{\delta}\right|_{\ell_{2}}^{2} \\
+\frac{\eta_{2}}{2}\left|\boldsymbol{\Psi}\left[\begin{array}{ll}
\hat{\boldsymbol{\alpha}}_{c}^{\mathrm{T}} & \hat{\boldsymbol{\alpha}}_{I}^{\mathrm{T}}
\end{array}\right]^{\mathrm{T}}-\hat{x}^{B}\right|_{\ell_{2}}^{2} .
\end{array}
$$


Given $\hat{\mathfrak{J}}^{k}$ and $\beta^{k}$, the sub-problem of (19) consists minimization of a strictly convex quadratic function that can be solved easily. Note that by setting the gradient of subproblem (19) to zero, its optimal solution is achieved as:

$$
\begin{aligned}
& \left(H^{\mathrm{T}} H+\delta W^{\mathrm{T}} W+\eta_{2} \boldsymbol{\Psi}^{\mathrm{T}} \boldsymbol{\Psi}\right) \hat{\boldsymbol{\alpha}}_{I}^{k+1}= \\
& \left(H^{\mathrm{T}} R_{I}+\eta_{2} \boldsymbol{\Psi}^{\mathrm{T}}\left(\hat{x}^{B}-\boldsymbol{\Psi} \hat{\boldsymbol{\alpha}}_{c}\right)+\frac{\delta}{2} W^{\mathrm{T}}\left(\hat{\mathfrak{J}}^{k}-\frac{\beta^{k}}{\delta}\right)\right) .
\end{aligned}
$$

Because of matrix inverse operation in (20), it is costly to directly minimize the quadratic function. Hence, in order to avoid the high computational complexity in (20), the steepest descent method with the optimal step is utilized in our implementation as follows:

$$
\begin{aligned}
\hat{\boldsymbol{\alpha}}_{I}^{k+1} & =\hat{\boldsymbol{\alpha}}_{I}^{k}-\mu^{k} \mathscr{G}^{k}, \mu^{k}>0 . \\
\mu & =\left|\frac{\mathscr{G}^{\mathrm{T}} \mathscr{G}}{\mathscr{G}^{\mathrm{T}}\left(H^{\mathrm{T}} H+\delta W^{\mathrm{T}} W+\eta_{2} \boldsymbol{\Psi}^{\mathrm{T}} \boldsymbol{\Psi}\right) \mathscr{G}}\right|
\end{aligned}
$$

where $\mu$ and $\mathscr{G}$ are the optimal step size and the gradient direction of the objective function [26]. In order to efficiently solve (17), similarly the sub-problem can be rewritten as:

$$
\begin{aligned}
\hat{\mathfrak{J}}^{k+1}=\arg \min _{\hat{\mathfrak{I}}} \eta_{1}|\hat{\mathfrak{I}}|_{\ell_{1}}+\left\langle\beta^{k}, \hat{\mathfrak{I}}-W \hat{\boldsymbol{\alpha}}_{I}^{k+1}\right\rangle \\
+\frac{\delta}{2}\left|\hat{\mathfrak{I}}-W \hat{\boldsymbol{\alpha}}_{I}^{k+1}\right|_{\ell_{2}}^{2} \\
=\arg \min _{\hat{\mathfrak{I}}} \eta_{1}|\hat{\mathfrak{I}}|_{\ell_{1}}+\frac{\delta}{2}\left|\hat{\mathfrak{I}}-W \hat{\boldsymbol{\alpha}}_{I}^{k+1}+\frac{\beta^{k}}{\delta}\right|_{\ell_{2}}^{2} .
\end{aligned}
$$

Given $\hat{\boldsymbol{\alpha}}_{I}^{k+1}$ and $\beta^{k}$, the soft-thresholding function [27] can be utilized as:

$$
\hat{\mathfrak{J}}^{k+1}=\mathscr{S}_{\frac{\eta_{1}}{\delta}}\left(W \hat{\boldsymbol{\alpha}}_{I}^{k+1}-\frac{\beta^{k}}{\delta}\right)
$$

where $\mathscr{S}(\Gamma, \xi)$ is a soft-thresholding function as

$$
\mathscr{S}(\Gamma, \xi)_{i}= \begin{cases}0, & \text { if }\left|\Gamma_{i}\right|<\xi \\ \frac{\Gamma_{i}}{\left|\Gamma_{i}\right|}\left(\left|\Gamma_{i}\right|-\xi\right), & \text { if }\left|\Gamma_{i}\right| \geq \xi\end{cases}
$$

\subsection{Summary of the Proposed Algorithm}

The proposed compressed sensing data acquisition method with joint signal ensemble recovery based on an iterative re-weighted $\ell_{1}$-minimization and a $\ell_{2}$ regularization is summarized in Algorithm 1. At each time slot $\tau \geq 1$, the fusion center periodically gathers the compressed sensing measurements in a data window by acquiring the reading from a set of the sensor nodes. Then, the sparsity constraint (i.e., by minimizing the $\ell_{1}$ norm) and $\ell_{2}$ regularization are incorporated in a single optimization formulation as (14). The optimization problem can be solved via an alternate direction method of multipliers implementation, resulting in an estimate of sensors signals. Once the algorithmic termination criteria are satisfied, i.e., difference of recovered sensors signals for two successive iterations $\left|W^{(n+1)}(\tau)-W^{(n)}(\tau)\right|_{\ell_{2}} /\left|W^{(n)}(\tau)\right|_{\ell_{2}}$ is smaller than a predefined threshold or there is a certain number of iterations (i.e., $n=n_{\max }$ ), then temporal step of the algorithm is moved one step forward, i.e., the data window moves one step and the recovery algorithm is processed again.

Algorithm 1 A Modified Compressed Sensing-Based Recovery Algorithm

Parameters: $c, L, \epsilon, \alpha$ and $n(\tau)$

\section{Initializations}

$a$ : Set $\tau=t-L+1$,

$b$ : Obtain $\left[\mathbf{x}_{1}(\tau), \mathbf{x}_{2}(\tau), \ldots, \mathbf{x}_{J}(\tau)\right]$, and form $\mathbf{X}(\tau)$

$c: \alpha_{c}=\alpha_{c_{\mathrm{opt}}}$ and compute $\mathbf{x}_{c}(\tau)=D \alpha_{c}$

\section{CS Measurements}

a: Compute $\boldsymbol{y}_{c_{j}}(\tau)=\Phi_{j}(\tau) \mathbf{x}_{c}(\tau)$ and $\boldsymbol{y}_{\mathrm{in}_{j}}(\tau)=\Phi_{j}(\tau)\left(\mathbf{x}_{j}(\tau)-D \alpha_{c}\right)$.

$b$ : Deliver the CS measurements $\boldsymbol{y}_{c_{j}}(\tau)$ and $\boldsymbol{y}_{\mathrm{in}_{j}}(\tau)$ in (4) to Fusion Center utilizing two different CDMA codes.

\section{Estimation of the Coefficients}

$a$ : Construct $\mathbf{R}(\tau), \Phi(\tau)$ and $\boldsymbol{\Psi} \hat{\alpha}(\tau)$.

$b$ : Solve (11) to obtain $\hat{\boldsymbol{\alpha}}(\tau)=\left[\hat{\boldsymbol{\alpha}}_{c}^{\mathrm{T}}(\tau) \cdots \hat{\boldsymbol{\alpha}}_{\mathrm{in}_{J}}^{\mathrm{T}}(\tau)\right]^{\mathrm{T}}$ as follows:

$b-1$ : Set $k=0, \beta^{0}=0, \hat{\boldsymbol{\alpha}}_{I}^{0}=0, \hat{\mathfrak{J}}^{0}=0$.

while: $\left|\hat{\boldsymbol{\alpha}}_{I}^{(k+1)}(\tau)-\hat{\boldsymbol{\alpha}}_{I}^{(k)}(\tau)\right|_{\ell_{2}} /\left|\hat{\boldsymbol{\alpha}}_{I}(\tau)\right|_{\ell_{2}} \epsilon$ or $k=k_{\max }$

$b-2: k=k+1$

$b$-3: compute $\hat{\boldsymbol{\alpha}}_{I}^{k+1}$ via the optimization problem (16)

$b$-4: compute $\hat{\mathfrak{J}}^{k+1}$ via the optimization problem (17)

$b$-5: compute $\hat{\beta}^{k+1}$ via the optimization problem (18)

\section{Recover the Signal Ensemble}

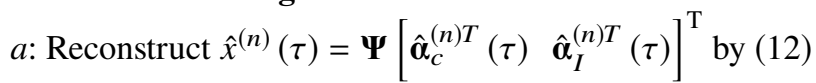

$b$ : Update the weights $\left(w_{i}\right)^{(n+1)}(\tau)=\left(\hat{\boldsymbol{\alpha}}_{i n_{i}}^{(n)}(\tau)+\epsilon_{0}\right)^{-1}$,

$i=1, \cdots, L J$.

$c$ : Terminate the algorithm when $\left|W^{(n+1)}(\tau)-W^{(n)}(\tau)\right|_{\ell_{2}} /\left|W^{(n)}(\tau)\right|_{\ell_{2}}$ is very small or $n=n_{\max }$.

$d$ : Report $\hat{\mathbf{X}}(\tau)$ as the reconstructed signal at time instant $\tau$. $e$ : step forward the temporal step of the algorithm $\tau=\tau+1$. And go to Initializations 


\section{Experimental Results}

In order to evaluate the reconstruction performance of the proposed method and compare it with the state of the art methods, experimental results are performed on the real temperature data collected once every 31 seconds from 54 Mica2Dot sensors deployed in the Intel Berkeley Research lab [28]. The signals $\mathbf{x}_{j} \in R^{400}, j=\{1,2, \ldots, 54\}$ from the 54sensors are sparse in a discrete wavelet transform (DWT) dictionary $D \in R^{400 \times 512}$ with different sparsity levels (Maximum 50-sparse). Specifically, the sensed signals are measured by Gaussian random matrices $\Phi_{j} \in R^{M_{x} \times 400}$ in each time instant $\tau=31 \mathrm{~s}$, and the sink received the measured signals. The simulation results are reported from the obtained mean results of 100 frames with different $\mathbf{x}_{j}$ s. The performance of the proposed reconstruction algorithm is compared with gradient-CS [11], SFAR-2D [12], reweightedlaplace [13], sequential-CS [20], modified-CS [29] and regularized modified-BPDN [30]. During the simulation, several parameters of the algorithms have been carefully tuned to perform an impartial comparison between the algorithms. All the experiments were implemented in MATLAM 2013a, on a Intel core i7 CPU with 16GB RAM and $3.5 \mathrm{GHz}$ processor. Moreover, in order to measure the performance of the different algorithms, we define normalized mean squared (NMS) error as

$$
N M S=\frac{1}{J} \sum_{j=1}^{J} \sum_{n=1}^{L}\left(\frac{\widehat{\mathbf{x}}_{j}(n)}{\left|\widehat{\mathbf{x}}_{j}\right|_{2}}-\frac{\mathbf{x}_{j}(n)}{\left|\mathbf{x}_{j}\right|_{2}}\right)^{2}
$$

where $\widehat{\mathbf{x}}_{j}, J$ and $L$ denote the estimate of $\mathbf{x}_{j}$, the number of sensors and the length of signals' samples, respectively.

The reconstruction signal to noise ratio (R-SNR); which is expressed as $R-S N R=10 \log _{10}\left(\frac{\left|\mathrm{x}_{j}\right|_{\ell_{2}}^{2}}{\left|\mathrm{x}_{j}-\widehat{\mathrm{x}}_{j}\right|_{\ell_{2}}^{2}}\right)$. And compression ratio $(\mathrm{CR})$ is the number of the original signal bits to the compressed signal.

Figure 1 depicts the average recovery performance of the different CS methods with respect to the different number of measurements $M_{x}$. As the proposed algorithm utilize fast ADMM solution, its reconstruction performance in the lower number of measurements is noticeably better than competitive state of the art algorithm. And as the number of measurements increases, the performance of the methods gradually approaches that of the proposed algorithm. The reconstruction times versus different measurement numbers for gradient-CS [11], SFAR-2D [12], reweighted-laplace [13], sequential-CS [20], modified-CS [29], regularized modifiedBPDN [30] and the proposed algorithm is depicted in Fig. 2. The consuming time for the algorithms is close to each other in a few number of the measurement samples, but as the number of samples increases, the simulation run time of our proposed method gradually changes. Compared to especially sequential-CS [20] and modified-CS [29], our algorithm requires less reconstruction time at the higher number of measurements.
The reconstruction performance of the algorithms against the additive white Gaussian (AWGN) channels is evaluated in different bit error (BER) rates. In this regard, the normalized mean squared error of the signal recovery for different bit error rates is shown in Fig. 3. Obviously, it can be inferred that modified-CS and gradient-CS unlike the other algorithms, result in a higher BERs with a worse recovery performance. The performance of SFAR-2D, reweightedlaplace and sequential-CS is closer to each other. More clearly, higher BERs bring more errors into the methods; however, the proposed recovery algorithm can significantly reconstruct signals while producing lower recovery BERs. The receiver operating characteristic (ROC) curve is an established means with which evaluate the sensing system's performance. These ROC curves depict the probability of detection $\left(P_{\mathrm{d}}\right)$ versus the probability of having a false alarm $\left(P_{\mathrm{d}}\right)$. Figure 4 shows the achieved ROC curves of the system performance for the different methods. It demonstrates that the proposed algorithm has close reconstruction performance to reweighted-laplace, but noticeably comparable to the other methods.

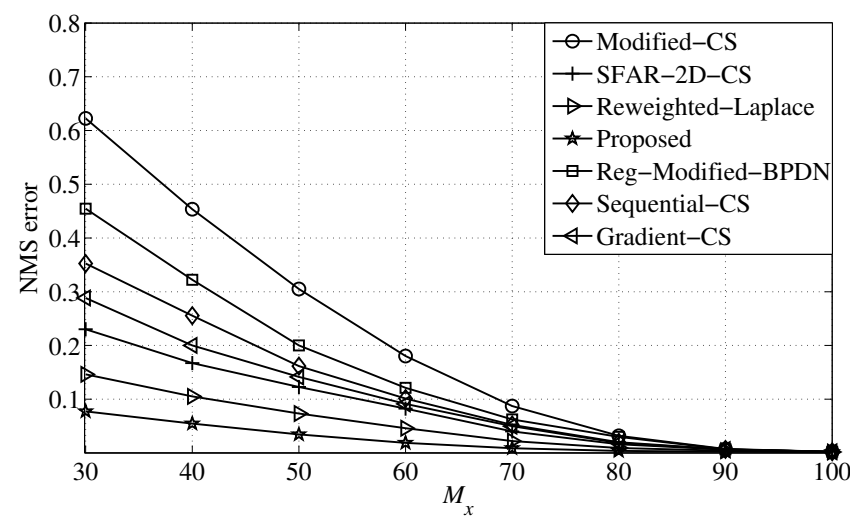

Fig. 1. The reconstruction performance of Modified-CS, regularized Modified-BPDN, sequential-CS and the proposed algorithm for data window size $L=400$ and $J=54$ with respect to the different measurement numbers.

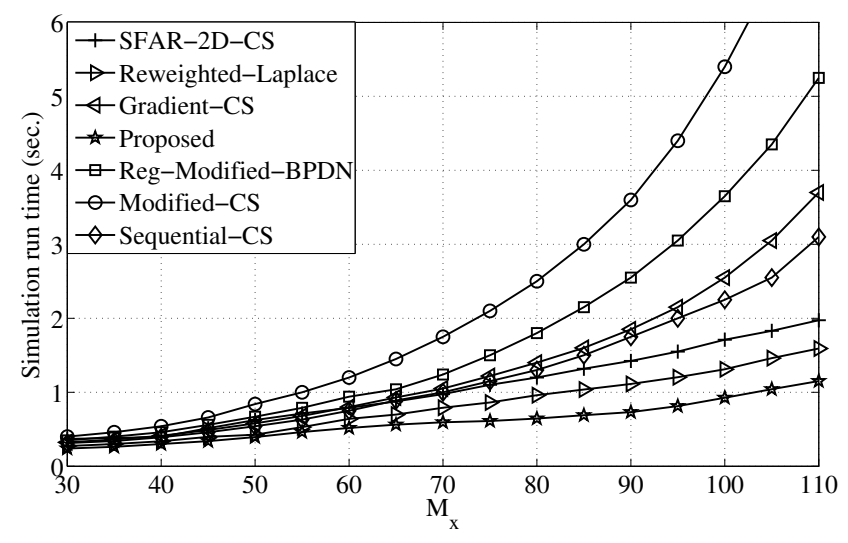

Fig. 2. The simulation run time of gradient-CS, SFAR-2D, reweighted-laplace, sequential-CS, modified-CS, regularized modified-BPDN and the proposed algorithm for data window size $L=400$ and $J=54$ with respect to the different measurement numbers. 


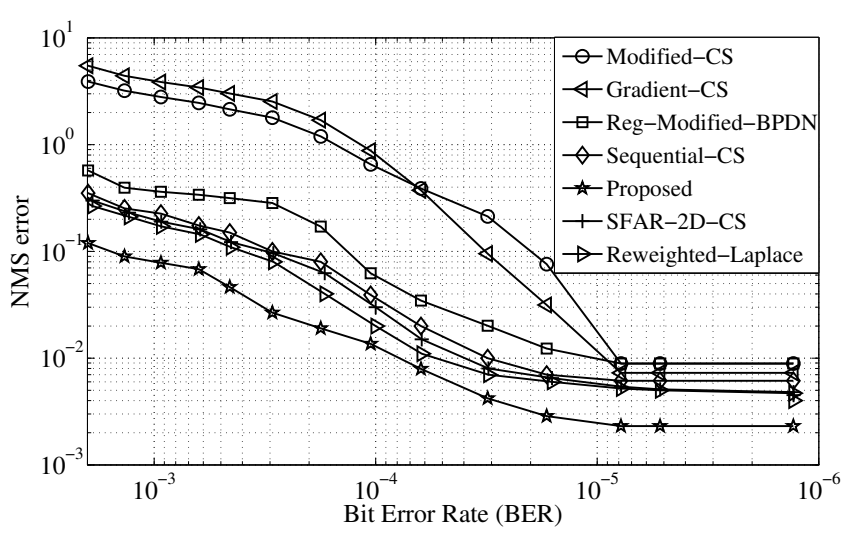

Fig. 3. The recovery performance of gradient-CS, SFAR-2D, reweighted-laplace, sequential-CS, modified-CS, regularized modified-BPDN and the proposed algorithm for data window size $L=400$ and $J=54$ with respect to different bit error rates (BERs).

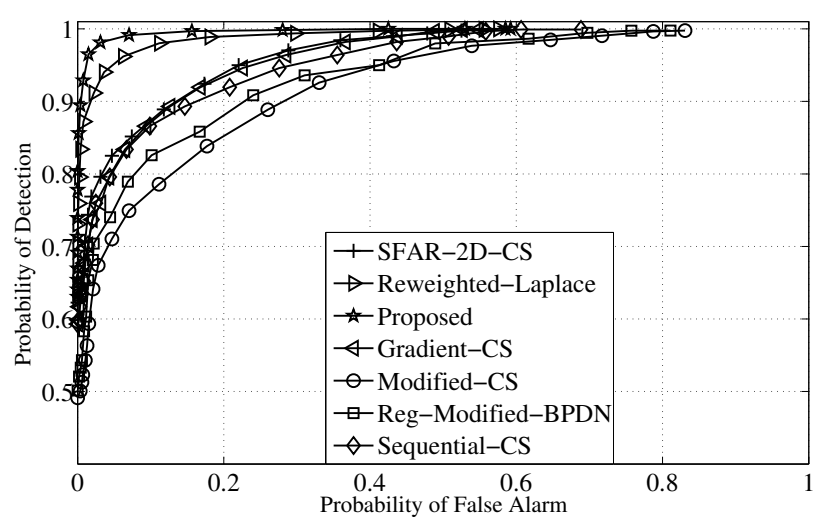

Fig. 4. ROC curves of gradient-CS, SFAR-2D, reweightedlaplace, sequential-CS, modified-CS, regularized modified-BPDN and the proposed algorithm.

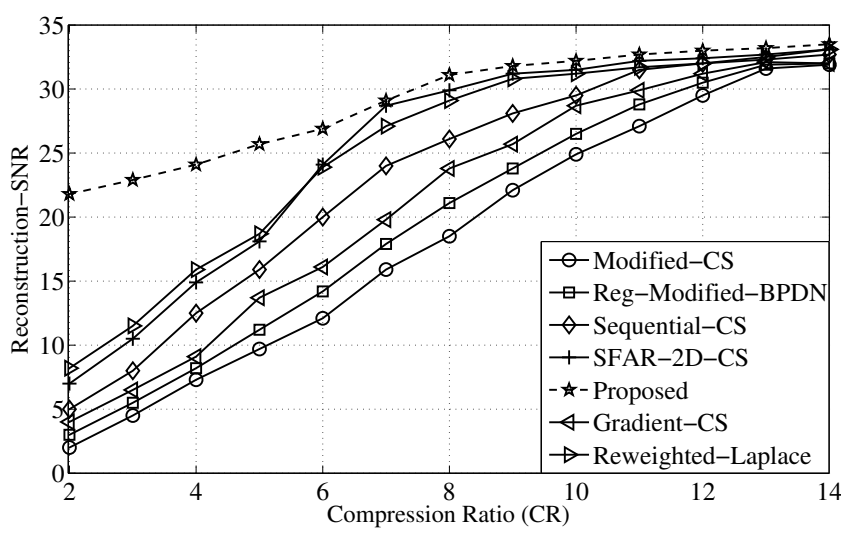

Fig. 5. Reconstruction-SNR of the recovered signals using the state of the art CS-based algorithms and the proposed algorithm with respect to different compression ratios $(\mathrm{CR})$.
In order to evaluate the quality of the reconstructed signals, the mean value of the reconstruction signal to noise ratio (R-SNR) versus several different compression ratios is depicted in Fig. 5. Since the purpose of this evaluation is to investigate the reconstruction capability of the proposed algorithm in comparison with other competitive methods, the signal sampling and recovery process were taken into account in the encoding-decoding scheme. Furthermore, the operations such as redundancy removal, quantization, and entropy coding were not applied. As it is shown in Fig. 5, the proposed method performs better signal reconstruction than the other methods in terms of several different compression ratios. The performance of the proposed algorithm is improved by utilizing not only the simultaneous extraction of spatiotemporal correlations within the signals in the data window, but also by efficiently solving the reconstruction problem via the introduced ADMM style implementation. It also shows that at a certain performance level, the proposed algorithm required fewer number of signal measurements to stage the same reconstruction performance as of other methods.

\section{Conclusion}

In this paper, a compressed sensing (CS) acquisition and joint recovery of spatiotemporal correlated signals method is proposed for efficient data gathering and accurate data streams reconstruction. More specifically, a method based on the alternating direction method of multipliers (ADMM) algorithm is developed due to its suitability in efficiently solving the large-scale convex optimization problems, ease of implementation and relatively fast.The experiments verify the effectiveness of the proposed algorithm compared with the state of the art methods.

\section{References}

[1] DONOHO, D. L. Compressed sensing. IEEE Transaction on Information Theory, 2006, vol. 52, no. 4, p. 1289-1306. DOI: 10.1109/TIT.2006.871582

[2] JAHANSHAHI, J. A., ESLAMI, M., GHORASHI, S. A. PSD map construction scheme based on compressive sensing in cognitive radio networks. IEICE Transaction on Communications, 2012, vol. 95, no. 4, p. 1056-1065. DOI: 10.1587/transcom.E95.B.1056

[3] JAHANSHAHI, J. A., ESLAMI, M., GHORASHI, S. A. Compressed sensing based dynamic PSD map construction in cognitive radio networks. Radioengineering, 2013, vol. 22, no. 2, p. 526-535. ISSN: $1805-9600$

[4] GUI, G., MEHBODNIYA, A., ADACHI, F. Sparse LMS/F algorithms with application to adaptive system identification. Wireless Cоттиnications and Mobile Computing, 2015, vol. 15, no. 12, p. 1649-1658. DOI: $10.1002 / \mathrm{wcm} .2453$

[5] GU, Y., JIN, J., MEI, S. $l_{0}$ norm constraint LMS algorithm for sparse system identification. IEEE Signal Processing Letters, 2009, vol. 16, no. 9, p. 774-777. DOI: 10.1109/LSP.2009.2024736

[6] HONG, X., JUBIN, G., CHEN, S. Zero-attracting recursive least squares algorithms. IEEE Transactions on Vehicular Technology, 2017, vol. 66, no. 1, p. 213-221. DOI: 10.1109/TVT.2016.2533664 
[7] LI, Y., WANG, Y., ALBU, F. Sparse channel estimation based on a reweighted least-mean mixed-norm adaptive filter algorithm. In Proceedings of the 24th European Signal Processing Conference (EUSIPCO). Budapest (Hungary), 2016, p. 2380-2384. DOI: $10.1109 /$ EUSIPCO.2016.7760675

[8] LI, Y., JIANG, Z., MOHAMMED, O., et al. Mixed norm constrained sparse APA algorithm for satellite and network echo channel estimation. IEEE Access, 2018, vol. 6, p. 65901-65908. DOI: 10.1109/ACCESS.2018.2878310

[9] LI, Y., JIANG, Z., SHI, W., HAN, X., CHEN, B. Blocked maximum correntropy criterion algorithm for cluster-sparse system identifications. IEEE Transactions on Circuits and Systems II: Express Briefs, 2019, p. 1-5. DOI: 10.1109/TCSII.2019.2891654

[10] CHENG, J., JIANG, H., MA, X., et al. Efficient data collection with sampling in WSNs: Making use of matrix completion techniques. In Proceedings of the IEEE Global Telecommunications Conference (GLOBECOM). Miami (USA), 2010, p. 1-5. DOI: $10.1109 /$ GLOCOM.2010.5684139

[11] STANKOVIC, L., DAKOVIC, M., VUJOVIC, S. Adaptive variable step algorithm for missing samples recovery in sparse signals. IET Signal Processing, 2014, vol. 8, no. 3, p. 246-256. DOI: 10.1049/iet-spr.2013.0385

[12] STANKOVIC, S., OROVIC, I. An approach to 2D signals recovering in compressive sensing context. Circuits, Systems, and Signal Processing, 2017, vol. 36, no. 4, p. 1700-1713. DOI: $10.1007 / \mathrm{s} 00034-016-0366-8$

[13] JIANG, T., ZHANG, X.W., LI, Y. Bayesian compressive sensing using reweighted laplace priors. AEU-International Journal of Electronics and Communications, 2018, vol. 97, p. 178-184. DOI: $0.1016 / \mathrm{j}$.aeue.2018.10.005

[14] AKYILDIZ, I. F., VURAN, M. C., AKAN, O. B. On exploiting spatial and temporal correlation in Wireless Sensor Networks. In Proceedings of the Conference on Modeling and Optimization in Mobile, Ad Hoc and Wireless Networks (WiOpt). Cambridge (UK), 2004, p. 71-80. DOI: 10.1016/j.procs.2013.09.025

[15] BARON, D., WAKIN, M. B., DUARTE, M., et al. Distributed compressed sensing. Preprint, arXiv:0901.3403v1, 2006, p. 1-42. Available at: http://arxiv.org/pdf/0901.3403v1.pdf

[16] DURATE, M. F., WAKIN, M. B., BARON, D., et al. Measurement bounds for sparse signal ensembles via graphical models. IEEE Transactions on Information Theory, 2013, vol. 59, no. 7, p. 4280-4289. DOI: 10.1109/TIT.2013.2252051

[17] MASOUM, A., MERATNIA, N., HAVINGA, P. A distributed compressive sensing technique for data gathering in wireless sensor networks. Procedia Computer Science, 2013, vol. 21, p. 207-216. DOI: $10.1016 /$ j.procs.2013.09.028

[18] QUER, G., MASIERO, R., PILlONETTO, G., et al. Sensing, compression, and recovery for WSNs: Sparse signal modeling and monitoring framework. IEEE Transactions on Wireless Communications, 2012, vol. 11, no. 10, p. 3447-3461. DOI: 10.1109/TWC.2012.081612.110612

[19] ASIF, M. S., ROMBERG, J. Sparse recovery of streaming signals using $\ell_{1}$-homotopy. IEEE Transactions on Signal Processing, 2014, vol. 62, no. 16, p. 4209-4223. DOI: 10.1109/TSP.2014.2328981

[20] Leinonen, M., CODREANU, M., JUNTTI, M. Sequential compressed sensing with progressive signal reconstruction in wireless sensor networks. IEEE Transactions on Wireless Communications, 2015, vol. 14, no. 3, p. 1622-1635. DOI: 10.1109/TWC.2014.2371017
[21] CANDES, E. J., WAKIN, M. B., BOYD, S. P. Enhancing sparsity by reweighted $\ell_{1}$ minimization. Journal of Fourier Analysis and Applications, 2008, vol. 14, no. 5, p. 877-905. DOI: $10.1007 / \mathrm{s} 00041-008-9045-\mathrm{x}$

[22] JAHANSHAHI, J. A., DANYALI, H., HELFROUSH, M. S. A distributed compressed sensing-based algorithm for the joint recovery of signal ensemble. Radioengineering, 2018, vol. 27, no. 2, p. 587-594. DOI: $10.13164 / \mathrm{re} .2018 .0587$

[23] PARIKH, N., BOYD, S. Proximal algorithms. Foundations and Trends in Optimization, 2014, vol. 1, no. 3, p. 127-239. DOI:10.1561/2400000003

[24] CHENG, J., YE, Q., JIANG, H., et al. STCDG: An efficient data gathering algorithm based on matrix completion for wireless sensor networks. IEEE Transactions on Wireless Communications, 2013, vol. 12, no. 2, p. 850-861. DOI: 10.1109/TWC.2012.121412.120148

[25] BLUMENSATH, T., DAVIES, M. E. Iterative thresholding for sparse approximations. Journal of Fourier Analysis and Applications, 2008, vol. 14, no. 5, p. 629-654. DOI: 10.1007/s00041-008-9035-z

[26] ESLAHI, N., AGHAGOLZADEH, A., ANDARGOLI, S.M.H. Image/video compressive sensing recovery using joint adaptive sparsity measure. Neurocomputing, 2016, vol. 200, p. 88-109. DOI: $10.1016 /$ j.neucom.2016.03.013

[27] DAUBECHIES, I., DEFRISE, M., DE MOL, C. An iterative thresholding algorithm for linear inverse problems with a sparsity constraint. Communications on Pure and Applied Mathematics, 2004, vol. 57, no. 11 , p. 1413-1457. DOI: $10.1002 /$ cpa.20042

[28] INTEL BERKELEY RESEARCH LAB. Intel Lab Data. Real sensors' readings from intel berkeley research lab. Available at: http://db.csail.mit.edu/labdata/labdata.html

[29] VASWANI, N., LU, W. Modified-CS: Modifying compressive sensing for problems with partially known support. IEEE Transactions on Signal Processing, 2010, vol. 58, no. 9, p. 4595-4607. DOI: $10.1109 /$ TSP.2010.2051150

[30] LU, W., VASWANI, N. Regularized modified BPDN for noisy sparse reconstruction with partial erroneous support and signal value knowledge. IEEE Transactions on Signal Processing, 2012, vol. 60, no. 1, p. 182-196. DOI: 10.1109/TSP.2011.2170981

\section{About the Authors ...}

Javad Afshar JAHANSHAHI received his M.Sc. degrees in Electrical Engineering from Shahid Beheshti University, in 2010. Now, he is pursuing Ph.D. program at the Department of Telecommunication Engineering, Shiraz University of Technology. His main research interests are in wireless communications and signal processing.

Habibollah DANYALI received his B.Sc. and M.Sc. degrees in Electrical Engineering respectively from Isfahan University of Technology, Isfahan, Iran, in 1991 and Tarbiat Modarres University, Tehran, Iran, in 1993. From 1994 to 2000 he was with the Dept. of Electrical Engineering, University of Kurdistan, Sanandaj, Iran, as a lecturer. In 2004 he received his Ph.D. degree in Computer Engineering from the University of Wollongong, Australia. He is currently working as an associate professor with the Dept. of Electrical and Electronics Engineering, Shiraz University of Technology, Shiraz, Iran. His research interests include data hiding, medical image processing, scalable image and video coding and biometrics. 
Mohammad Sadegh HELFROUSH received the B.S. and M.S. degrees in Electrical Engineering from Shiraz University, Shiraz, and Sharif University of Technology, Tehran in 1993 and 1995, respectively. He performed his Ph.D. degree in Electrical Engineering from Tarbiat Modares University,
Tehran, Iran. He is working as an associate professor in the Dept. of Electrical and Electronics Engineering, Shiraz Univ. of Technology, Shiraz, Iran. His research interests include content-based image retrieval, pattern recognition and medical image processing. 